\title{
IsoGene: An R Package for Analyzing Dose-response Studies in Microarray Experiments
}

by Setia Pramana, Dan Lin, Philippe Haldermans, Ziv Shkedy, Tobias Verbeke, Hinrich Göhlmann, An De Bondt, Willem Talloen and Luc Bijnens.

\begin{abstract}
IsoGene is an $\mathrm{R}$ package for the analysis of dose-response microarray experiments to identify gene or subsets of genes with a monotone relationship between the gene expression and the doses. Several testing procedures (i.e., the likelihood ratio test, Williams, Marcus, the $M$, and Modified $M$ ), that take into account the order restriction of the means with respect to the increasing doses are implemented in the package. The inference is based on resampling methods, both permutations and the Significance Analysis of Microarrays (SAM).
\end{abstract}

\section{Introduction}

The exploration of dose-response relationship is important in drug-discovery in the pharmaceutical industry. The response in this type of studies can be either the efficacy of a treatment or the risk associated with exposure to a treatment. Primary concerns of such studies include establishing that a treatment has some effect and selecting a dose or doses that appear efficacious and safe (Pinheiro et al., 2006). In recent years, dose-response studies have been integrated with microarray technologies (Lin et al., 2010). Within the microarray setting, the response is gene expression measured at a certain dose level. The aim of such a study is usually to identify a subset of genes with expression levels that change with experimented dose levels.

One of four main questions formulated in doseresponse studies by Ruberg (1995a, 1995b) and Chuang-Stein and Agresti (1997) is whether there is any evidence of the drug effect. To answer this question, the null hypothesis of homogeneity of means (no dose effect) is tested against ordered alternatives. Lin et al. $(2007,2010)$ discussed several testing procedures used in dose-response studies of microarray experiments. Testing procedures which take into account the order restriction of means with respect to the increasing doses include Williams (Williams, 1971 and 1972), Marcus (Marcus, 1976), the likelihood ratio test (Barlow et al. 1972, and Robertson et al. 1988), the $M$ statistic (Hu et al., 2005) and the modified $M$ statistic (Lin et al., 2007).

To carry out the analysis of dose-response microarray experiments discussed by Lin et al. (2007,
2010), an R package called IsoGene has been developed. The IsoGene package implements the testing procedures described by Lin et al. (2007) to identify a subset of genes where a monotone relationship between gene expression and dose can be detected. In this package, the inference is based on resampling methods, both permutations (Ge et al., 2003) and the Significance Analysis of Microarrays (SAM), Tusher et al., 2001. To control the False Discovery Rate (FDR) the Benjamini Hochberg $(\mathrm{BH})$ procedure (Benjamini and Hochberg, 1995) is implemented.

This paper introduces the IsoGene package with background information about the methodology used for analysis and its main functions. Illustrative examples of analysis using this package are also provided.

\section{Testing for Trend in Dose Response Microarray Experiments}

In a microarray experiment, for each gene, the following ANOVA model is considered:

$$
Y_{i j}=\mu\left(d_{i}\right)+\varepsilon_{i j}, i=0,1, \ldots, K, j=1,2, \ldots, n_{i},
$$

where $Y_{i j}$ is the $j$ th gene expression at the $i$ th dose level, $d_{i}(i=0,1, \ldots, K)$ are the $K+\mathbf{1}$ dose levels, $\mu\left(d_{i}\right)$ is the mean gene expression at each dose level, and $\varepsilon_{i j} \sim N\left(0, \sigma^{2}\right)$. The dose levels $d_{0}, \ldots, d_{K}$ are strictly increasing.

The null hypothesis of homogeneity of means (no dose effect) is given by

$$
H_{0}: \mu\left(d_{0}\right)=\mu\left(d_{1}\right)=\cdots=\mu\left(d_{K}\right) .
$$

where $\mu\left(d_{i}\right)$ is the mean response at dose $d_{i}$ with $i=0, . ., K$, where $i=0$ indicates the control. The alternative hypotheses under the assumption of monotone increasing and decreasing trend of means are respectively specified by

$$
\begin{gathered}
H_{1}^{U p}: \mu\left(d_{0}\right) \leq \mu\left(d_{1}\right) \leq \cdots \leq \mu\left(d_{K}\right), \\
H_{1}^{\text {Down }}: \mu\left(d_{0}\right) \geq \mu\left(d_{1}\right) \geq \cdots \geq \mu\left(d_{K}\right) .
\end{gathered}
$$

For testing $H_{0}$ against $H_{1}^{\text {Down }}$ or $H_{1}^{U p}$, estimation of the means under both the null and alternative hypotheses is required. Under the null hypothesis, the estimator for the mean response $\hat{\mu}$ is the overall sample mean. Let $\hat{\mu}_{0}^{\star}, \hat{\mu}_{1}^{\star}, \ldots, \hat{\mu}_{K}^{\star}$ be the maximum likelihood estimates for the means (at each dose level) under the order restricted alternatives. Barlow et al. (1972) and Robertson et al. (1988) showed that 
$\hat{\mu}_{0}^{\star}, \hat{\mu}_{1}^{\star}, \ldots, \hat{\mu}_{K}^{\star}$ are given by the isotonic regression of the observed means.

In order to test $H_{0}$ against $H_{1}^{\text {Down }}$ or $H_{1}^{U p}$, Lin et al. (2007) discussed five testing procedures shown in Figure 1. The likelihood ratio test $\left(\bar{E}_{01}^{2}\right)$ (Bartholomew 1961, Barlow et al. 1972, and Robertson et al. 1988) uses the ratio between the variance under the null hypothesis $\left(\hat{\sigma}_{H_{0}}^{2}\right)$ and the variance under order restricted alternatives $\left(\hat{\sigma}_{H_{1}}^{2}\right)$ :

$$
\Lambda_{01}^{\frac{2}{N}}=\frac{\hat{\sigma}_{H_{1}}^{2}}{\hat{\sigma}_{H_{0}}^{2}}=\frac{\sum_{i j}\left(y_{i j}-\hat{\mu}_{j}^{\star}\right)^{2}}{\sum_{i j}\left(y_{i j}-\hat{\mu}\right)^{2}} .
$$

Here, $\hat{\mu}=\sum_{i j} y_{i j} / \sum_{i} n_{i}$ is the overall mean and $\hat{\mu}_{i}^{\star}$ is the isotonic mean of the $i$ th dose level. The null hypothesis is rejected for a "small" value of $\Lambda_{01}^{\frac{2}{N}}$. Hence, $H_{0}$ is rejected for a large value of $\bar{E}_{01}^{2}=1-\Lambda_{01}^{\frac{2}{N}}$.

\begin{tabular}{ll}
\hline Test statistic & Formula \\
\hline Likelihood Ratio & $\bar{E}_{01}^{2}=\frac{\sum_{i j}\left(y_{i j}-\hat{\mu}\right)^{2}-\sum_{i j}\left(y_{i j}-\hat{\mu}_{i}^{\star}\right)^{2}}{\sum_{i j}\left(y_{i j}-\hat{\mu}\right)^{2}}$ \\
Test (LRT) & $t=\left(\hat{\mu}_{K}^{\star}-\bar{y}_{0}\right) / s$ \\
Williams & $t=\left(\hat{\mu}_{K}^{\star}-\hat{\mu}_{0}^{\star}\right) / s$ \\
Marcus & $M=\left(\hat{\mu}_{K}^{\star}-\hat{\mu}_{0}^{\star}\right) / \tilde{s}$ \\
M & $M^{\prime}=\left(\hat{\mu}_{K}^{\star}-\hat{\mu}_{0}^{\star}\right) \tilde{s}^{\prime}$ \\
Modified M $\left(\mathrm{M}^{\prime}\right)$ & \\
\hline
\end{tabular}

Figure 1: Test statistics for trend test, where $s=$ $\sqrt{2 \times \sum_{i=0}^{K} \sum_{j=1}^{n_{i}}\left(y_{i j}-\hat{\mu}_{i}\right)^{2} /\left(n_{i}(n-K)\right)}$, $\tilde{s}=\sqrt{\sum_{i=0}^{K} \sum_{j=1}^{n_{i}}\left(y_{i j}-\hat{\mu}_{i}^{\star}\right)^{2} /(n-K)}$, $\tilde{s}^{\prime}=\sqrt{\sum_{i=0}^{K} \sum_{j=1}^{n_{i}}\left(y_{i j}-\hat{\mu}_{i}^{\star}\right)^{2} /(n-I)}$, and $I$ is the unique number of isotonic means.

Williams (1971, 1972) proposed a step-down procedure to test for the dose effect. The tests are performed sequentially from the comparison between the isotonic mean of the highest dose and the sample mean of the control, to the comparison between the isotonic mean of the lowest dose and the sample mean of the control. The procedure stops at the dose level where the null hypothesis (of no dose effect) is not rejected. The test statistic is shown in Figure 1 , where $\bar{y}_{0}$ is the sample mean at the first dose level (control), $\hat{\mu}_{i}^{\star}$ is the estimate for the mean at the $i$ th dose level under the ordered alternative, $n_{i}$ is the number of replications at each dose level, and $s^{2}$ is an estimate of the variance. A few years later, Marcus (1976) proposed a modification to Williams's procedure by replacing the sample mean of the control dose $\left(\bar{y}_{0}\right)$ with the isotonic mean of the control dose $\left(\hat{\mu}_{0}^{\star}\right)$.

Hu et al. (2005) proposed a test statistic (denoted by $M$ ) that was similar to Marcus' statistic, but with the standard error estimator calculated under the ordered alternatives. This is in contrast to Williams' and Marcus' approaches that used the within group sum of squares under the unrestricted means. Moreover, $\mathrm{Hu}$ et al. (2005) used $n-K$ as the degrees of freedom. However, the unique number of isotonic means is not fixed, but changes across genes. For that reason, Lin et al. (2007) proposed a modification to the standard error estimator used in the $M$ statistic by replacing it with $(n-I)$ as the degrees of freedom, where $I$ is the unique number of isotonic means for a given gene. The five test statistics discussed above are based on the isotonic regression of the observed means. Estimation of isotonic regression requires the knowledge of the direction of the trend (increasing/decreasing). In practice, the direction is not known in advance. Following Barlow et al. (1972), the IsoGene package calculates the likelihood of the isotonic trend for both directions and chooses the direction for which the likelihood is maximized.

The Significance Analysis of Microarrays procedure (SAM, Tusher et al., 2001) can be also adapted to the five test statistics described above. The generic algorithm of SAM discussed by Chu et al. (2001) is implemented in this package. For the $t$-type test statistics (i.e., Williams, Marcus, the $M$, and the $M^{\prime}$ ), a fudge factor is added in the standard error of the mean difference. For example, the SAM regularized test statistic for the $M^{\prime}$ is given by,

$$
M^{\prime S A M}=\frac{\hat{\mu}_{K}^{\star}-\hat{\mu}_{0}^{\star}}{\tilde{s}^{\prime}+s_{0}},
$$

where $s_{0}$ is the fudge factor and is estimated from the percentiles of standard errors of all genes which minimize the Coefficient of Variation (CV) of the Median Absolute Deviation (MAD) of the SAM regularized test statistics. For the F-type test statistic, such as $\bar{E}_{01}^{2}$, the SAM regularized test statistic is defined by,

$$
\bar{E}_{01}^{2 S A M}=\frac{\sqrt{\hat{\sigma}_{H_{0}}^{2}-\hat{\sigma}_{H_{1}}^{2}}}{\sqrt{\hat{\sigma}_{H_{0}}^{2}}+s_{0}} .
$$

\section{Multiplicity}

In this study, a gene-by-gene analysis is carried out. When many hypotheses are tested, the probability of making the type I error increases sharply with the number of hypotheses tested. Hence, multiplicity adjustments need to be performed. Dudoit et al. (2003) and Dudoit and van der Laan (2008) provided extensive discussions on the multiple adjustment procedures in genomics. Lin et al. (2007) compared several approaches for multiplicity adjustments, and showed the application in dose-response microarray experiments. In the IsoGene package the inference is based on resampling-based methods. The Benjamini Hochberg $(\mathrm{BH})$ procedure is used to control the FDR . We use the definition of FDR from Benjamini and Hochberg (1995). The Significance Analysis of Microarrays (SAM, Tusher et al., 2001) approach is also 
considered, which estimates the FDR by using permutations, where the FDR is computed as median of the number of falsely called genes divided by the number of genes called significant.

\section{Introduction to IsoGene Package}

The IsoGene package provides the estimates and $p$-values of five test statistics for testing for monotone trends discussed in the previous section. The $p$-values are calculated based on a resampling procedure in which the distribution of the statistic under the null hypothesis is approximated using permutations.

The main functions of the IsoGene package are IsoRawp () and IsoTestBH () which calculate the raw $p$-values using permutations and adjust them using the Benjamini-Hochberg (BH-FDR, Benjamini and Hochberg, 1995) and Benjamini-Yekutieli (BY-FDR, Benjamini and Yekutieli, 2001) procedures. The IsoGene package also implements the Significance Analysis of Microarrays (SAM) by using function IsoTestSAM ( ).

\begin{tabular}{|c|c|}
\hline Function & Description \\
\hline IsoRawp () & $\begin{array}{l}\text { Calculates raw p-values for } \\
\text { each test statistic using } \\
\text { permutations }\end{array}$ \\
\hline IsoTestBH () & $\begin{array}{l}\text { Adjusts raw p-values of the } \\
\text { five test statistics using the } \\
\text { BH- or BY-FDR procedure }\end{array}$ \\
\hline IsoGene1 () & $\begin{array}{l}\text { Calculates the values of the } \\
\text { five test statistics for a } \\
\text { single gene }\end{array}$ \\
\hline IsoGenem () & $\begin{array}{l}\text { Calculates the values of the } \\
\text { five test statistics for all genes }\end{array}$ \\
\hline IsoPlot () & $\begin{array}{l}\text { Plots the data points, sample } \\
\text { means at each dose and } \\
\text { an isotonic regression line } \\
\text { (optional) }\end{array}$ \\
\hline IsopvaluePlot () & $\begin{array}{l}\text { Plots the } p^{U p} \text { and } p^{D o w n} \text {-values } \\
\text { of a gene for a given test }\end{array}$ \\
\hline IsoBHplot () & $\begin{array}{l}\text { Plots the raw p-values and } \\
\text { adjusted BH- and BY-FDR } \\
p \text {-values }\end{array}$ \\
\hline IsoGenemSAM() & $\begin{array}{l}\text { Calculates the values for the } \\
\text { five SAM regularized test } \\
\text { statistics }\end{array}$ \\
\hline IsoTestSAM() & $\begin{array}{l}\text { Obtains the list of significant } \\
\text { genes using the SAM } \\
\text { procedure }\end{array}$ \\
\hline
\end{tabular}

Figure 2: The main IsoGene package functions.

The supporting functions are IsoGenem() and IsoGenemSAM(), which calculate the values for the five test statistics and for five SAM regularized test statistics, respectively. The functions IsopvaluePlot (), IsoBHplot(), and IsoPlot () can be used to display the data and show the results of the testing procedures. The summary of the functions and their descriptions are presented in Figure 2.

The IsoGene package can be obtained from CRAN: http:/ / cran.r-project.org/package=IsoGene. The IsoGene package requires the ff and Iso packages.

\section{Example 1: Data Exploration}

To illustrate the analysis of dose-response in microarray using IsoGene package, we use the dopamine data. The data were obtained from a pre-clinical evaluation study of an active compound (Göhlmann and Talloen, 2009). In this study the potency of the compound was investigated. The compound had 6 dose levels $(0,0.01,0.04,0.16,0.63,2.5 \mathrm{mg} / \mathrm{kg})$ and each dose was given to four to five independent rats. The experiment was performed using Affymetrix whole-human genome chip. There are 26 chips/arrays and each chip/array contains 11,562 probe sets (for simplicity, we refer to the probe sets as genes). The dopamine data set with 1000 genes is provided inside the package as example data. The complete data set can be obtained on request upon the first author. For this paper, the analysis is based on the original data set (using all genes).

The example data is in an Expressionset object called dopamine. More detailed explanation of the Expressionset object is discussed by Falcon et al. (2007). In order to load the object into $\mathbf{R}$, the following code can be used:

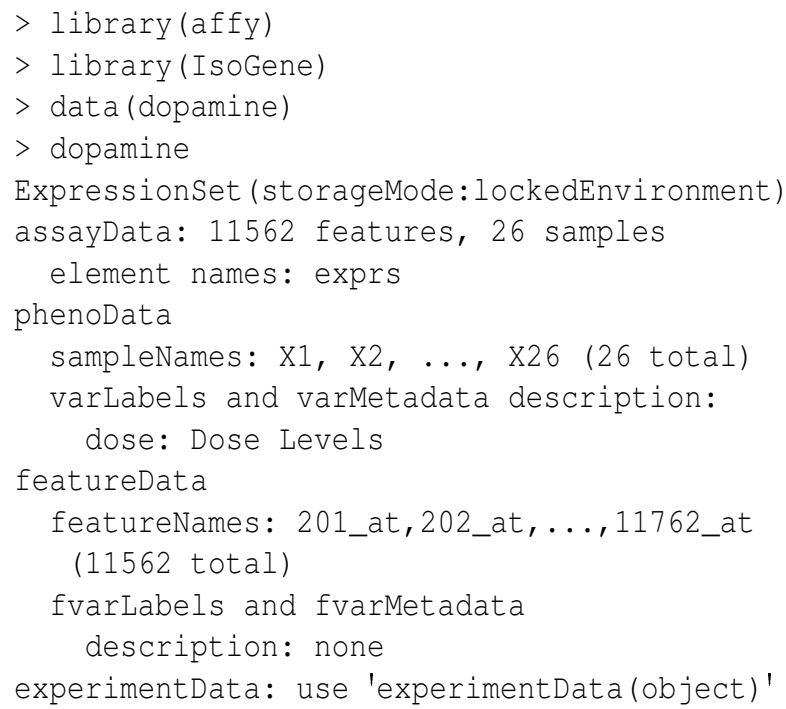

The IsoGene package requires the information of dose levels and gene expression as input. The information of dose levels and the $\log 2$ transformed gene intensities can be extracted using the following code:

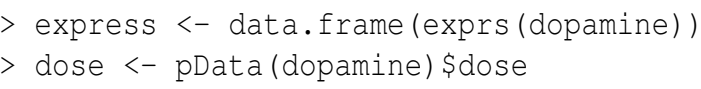

For data exploration, the function IsoPlot () can be used to produce a scatterplot of the data. The 
function IsoPlot () has two options to specify the dose levels, i.e., type ="ordinal" or "continuous". By default, the function produces the plot with continuous dose levels and data points. To add an isotonic regression line, one can specify add. curve=TRUE.

Plots of the data and an isotonic regression line for one of the genes in the data set with dose on the continuous and ordinal scale can be produced by using the following code:
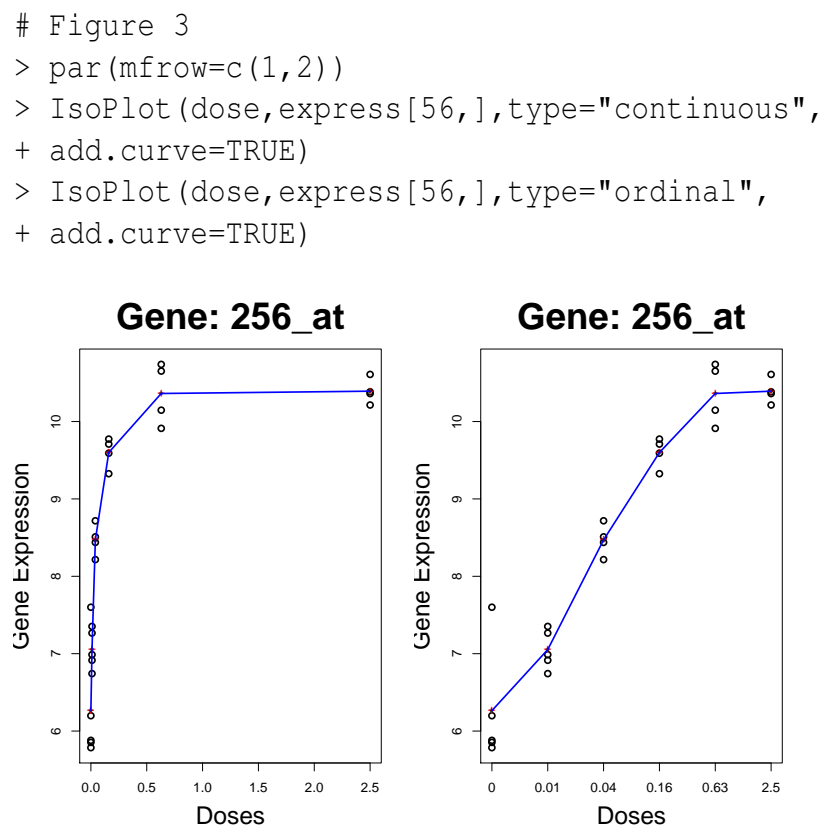

Figure 3: The plots produced by IsoP lot () with dose as continuous variable (left panel) and dose as ordinal variable (right panel and the real dose level is presented on the $\mathrm{x}$-axis). The data points are plotted as circles, while the sample means as red crosses and the fitted increasing isotonic regression model as a blue solid line.

\section{Example 2: Resampling-based Multiple Testing}

In this section, we illustrate an analysis to test for monotone trend using the five statistics presented in section 2 using the IsoGene package. The function IsoRawp () is used to calculate raw $p$-values based on permutations. The dose levels, the data frame of the gene expression, and the number of permutations used to approximate the null distribution need to be specified in this function. Note that, since the permutations are generated randomly, one can use function set. seed to obtain the same random number. To calculate raw $p$-values for the dopamine data using 1000 permutations with seed=1234, we can use the following code:

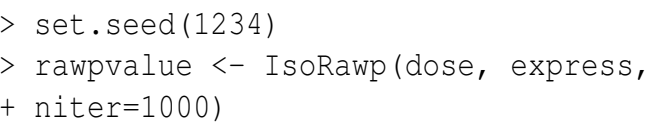

The output object rawpvalue is a list with four components containing the $p$-values for the five test statistics: two-sided $p$-values, one-sided $p$-values, $p^{U p}$-values and $p^{\text {Down }}$-values. The codes to extract the component containing two-sided $p$-values for the first four genes are presented below. In a similar way, one can obtain other components.

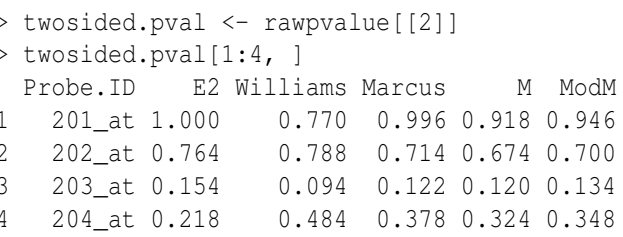

Once the raw $p$-values are obtained, one needs to adjust these $p$-values for multiple testing. The function IsoTestBH () is used to adjust the $p$-values while controlling for the FDR. The raw p-values (e.g., rawp ), FDR level, type of multiplicity adjustment (BHFDR or BY-FDR) and the statistic need to be specified in following way:

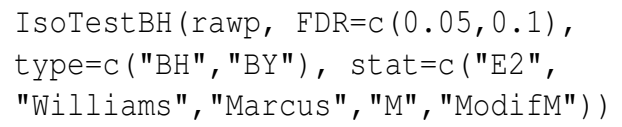

IsoTestBH () produces a list of genes, which have a significant increasing/decreasing trend. The following code can be used to adjust the two-sided $p$-values of the $\bar{E}_{01}^{2}$ using the BH-FDR adjustment:

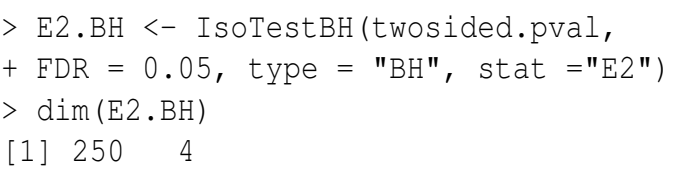

The object $\mathrm{E} 2 \mathrm{BH}$ contains a list of significant genes along with the probe ID, the corresponding row numbers of the gene in the original data set, the unadjusted (raw), and the BH-FDR adjusted $p$ values of the $\bar{E}_{01}^{2}$ test statistic. In the dopamine data, there are 250 genes tested with a significant monotone trend using the likelihood ratio test $\left(\bar{E}_{01}^{2}\right)$ at the 0.05 FDR level. The first four significant genes are listed below:

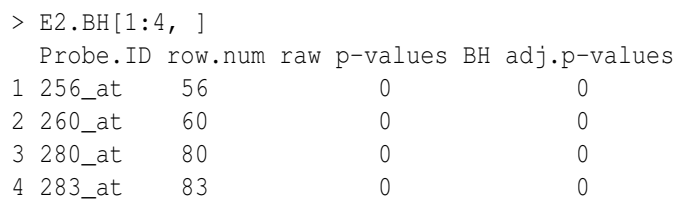

One can visualize the number of significant findings for the BH-FDR and BY-FDR procedures for a given test statistic using the IsoBHP lot () function.

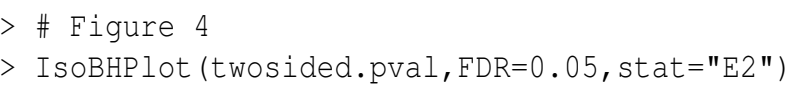

Figure 4 shows the raw $p$-values (solid blue line), the BH-FDR adjusted $p$-values (dotted and dashed red line) and BY-FDR (dashed green line) adjusted $p$ values for the $\bar{E}_{01}^{2}$. 


\section{E2: Adjusted $\mathrm{p}$ values by $\mathrm{BH}$ and $\mathrm{BY}$}

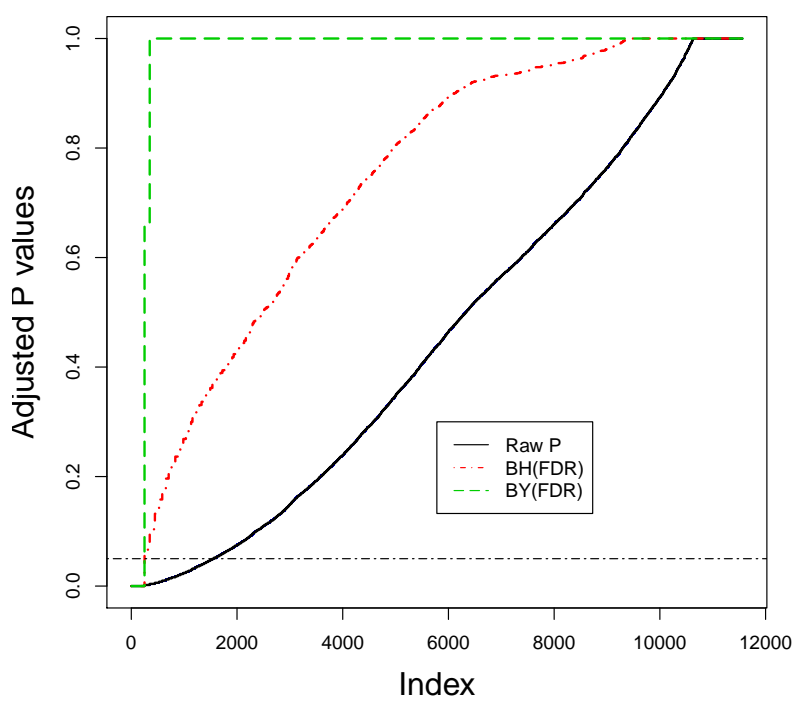

Figure 4: Plot of unadjusted, BH-FDR and BY-FDR adjusted p-values for $\bar{E}_{01}^{2}$

\section{Example 3: Significance Analysis of Dose- response Microarray Data (SAM)}

The Significance Analysis of Microarrays (SAM) for testing for the dose-response relationship under order restricted alternatives is implemented in the IsoGene package as well. The function IsoTestSAM() provides a list of significant genes based on the SAM procedure.

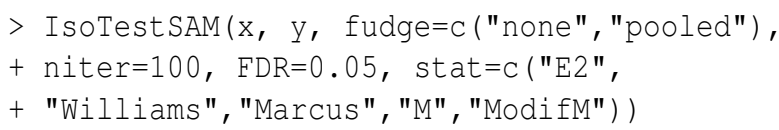

The input for this function is the dose levels (x), gene expression (y), number of permutations (niter), the FDR level, the test statistic, and the option of using fudge factor. The option fudge is used to specify the calculation the fudge factor in the SAM regularized test statistic. If the option fudge ="pooled" is used, the fudge factor will be calculated using the method described in the SAM manual (Chu et al., 2001). If we specify fudge $=$ "none" no fudge factor is used.

The following code is used for analyzing the dopamine data using the SAM regularized $M^{\prime}$-test statistic:

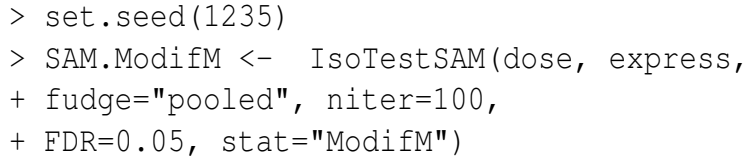

The resulting object SAM.ModifM, contains three components:

1. sign.genes 1 contains a list of genes declared significant using the SAM procedure.
2. qqs tat gives the SAM regularized test statistics obtained from permutations.

3. allfdr provides a delta table in the SAM procedure for the specified test statistic.

To extract the list of significant gene, one can do:

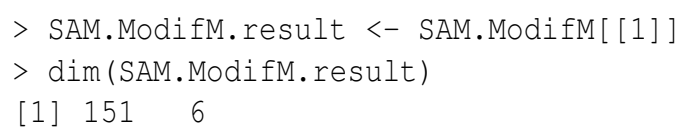

The object SAM.ModifM.result, contains a matrix with six columns: the Probe IDs, the corresponding row numbers of the genes in the data set, the observed SAM regularized $M^{\prime}$ test statistics, the $q$ values (obtained from the lowest False Discovery Rate at which the gene is called significant), the raw $p$-values obtained from the joint distribution of permutation test statistics for all of the genes as described by Storey and Tibshirani (2003), and the BHFDR adjusted $p$-values.

For dopamine data, there are 151 genes found to have a significant monotone trend based on the SAM regularized $M^{\prime}$ test statistic with the FDR level of 0.05. The SAM regularized $M^{\prime}$ test statistic values along with $q$-values for the first four significant genes are presented below.

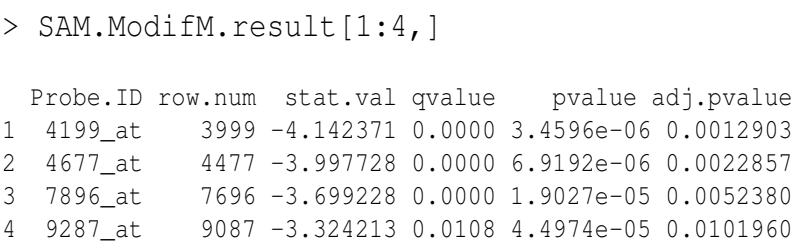

Note that genes are sorted increasingly based on the SAM regularized $M^{\prime}$ test statistic values (i.e., stat.val).

In the IsoGene package, the function IsoSAMPlot() provides graphical outputs of the SAM procedure. This function requires the SAM regularized test statistic values and the delta table in the SAM procedure, which can be obtained from the resulting object of the IsoTestSAM() function, which in this example data is called SAM. ModifM. To extract the objects we can use the following code:

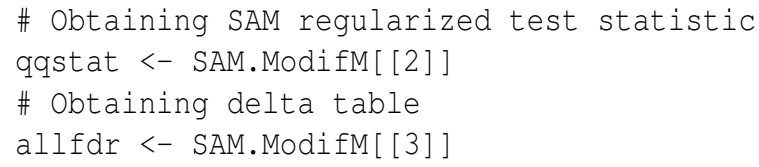

We can also obtain the qqstat and allfdr from the functions Isogqstat () and Isoallfdr(), respectively. The code for the two functions are:

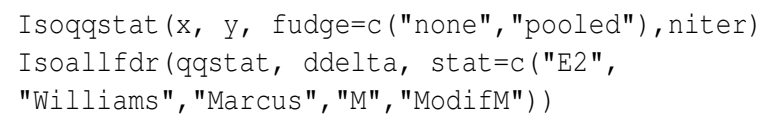

The examples of using the functions Isoqqstat () and Isoallfdr() are as follows: 
\# Obtaining SAM regularized test statistic

$>$ qqstat <- Isoqqstat (dose, express,

+ fudge="pooled", niter=100)

\# Obtaining delta table

$>$ allfdr <- Isoallfdr (qqstat, , stat="ModifM")

Note that in Isoallfdr (), ddelta is left blank, with default values taken from the data, i.e., all the percentiles of the standard errors of the $M^{\prime}$ test statistic.

The two approaches above will give the same result. Then to produces the SAM plot for the SAM regularized $M^{\prime}$ test statistic, we can use the function IsoSAMP lot:

\# Figure 5

$>$ IsoSAMPlot (qqstat, allfdr, $\mathrm{FDR}=0.05$,

+ stat $=$ "ModifM")
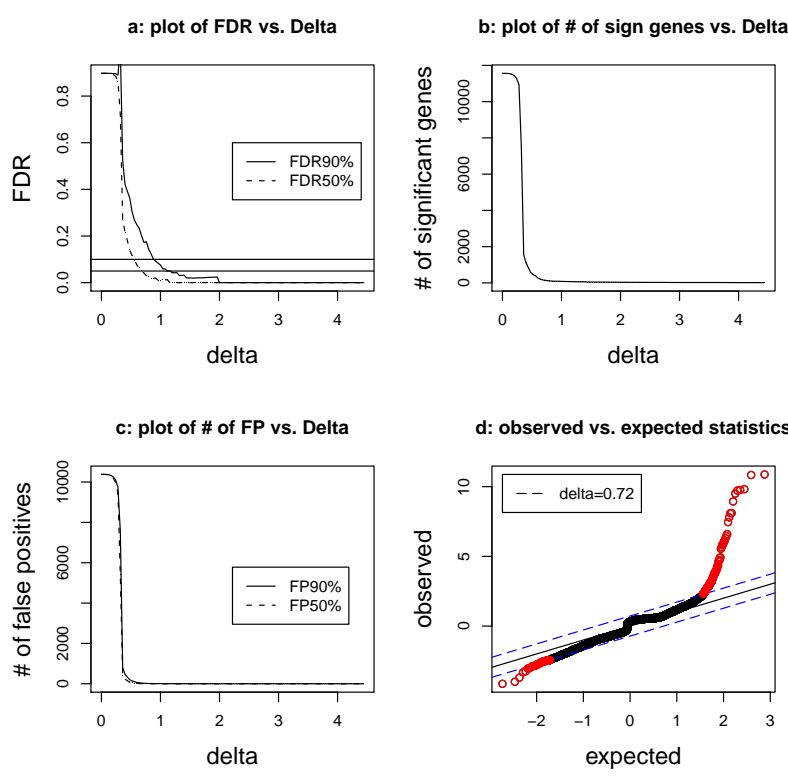

Figure 5: The SAM plots: a.Plot of the FDR vs. $\Delta ; b$. Plot of number of significant genes vs. $\Delta ; c$. Plot of number of false positives vs. $\Delta ; d$. Plot of the observed vs. expected test statistics.

Panel $a$ of Figure 5 shows the FDR (either 50\% or $90 \%$ (more stringent)) vs. $\Delta$, from which, user can choose the $\Delta$ value with the corresponding desired FDR. The FDR 50\% and FDR 90\% are obtained from, respectively, the median and 90th percentile of the number of falsely called genes (number of false positives) divided by the number of genes called significant, which are estimated by using permutations (Chu et al., 2001). Panel $b$ shows the number of significant genes vs. $\Delta$, and panel $c$ shows the number of false positives (either obtained from 50th or 90th percentile) vs. $\Delta$. Finally, panel $d$ shows the observed vs. the expected (obtained from permutations) test statistics, in which the red dots are those genes called differentially expressed.

\section{Comparison of the Results of Resampling-based Multiple Test- ing and the SAM}

In the previous sections we have illustrated analysis for testing a monotone trend for dose-response microarray data by using permutations and the SAM. The same approach can also be applied to obtain a list of significant genes based on other statistics and other multiplicity adjustments. Figure 6 presents the number of genes that are found to have a significant monotone trend using five different statistics with the FDR level of 0.05 using the two approaches.

It can be seen from Figure 6 that the $\bar{E}_{01}^{2}$ gives a higher number of significant genes than other $t$-type statistics. Adding the fudge factor to the statistics leads to a smaller number of significant genes using the SAM procedure for the $t$-type test statistics as compared to the BH-FDR procedure.

\begin{tabular}{l|c|c|c}
\hline \hline & \multicolumn{3}{|c}{ Number of significant genes } \\
Test statistic & BH-FDR & SAM & \# common genes \\
\hline $\bar{E}_{01}^{2}$ & 250 & 279 & 200 \\
Williams & 186 & 104 & 95 \\
Marcus & 195 & 117 & 105 \\
$M$ & 209 & 158 & 142 \\
$M^{\prime}$ & 203 & 151 & 134 \\
\hline \hline
\end{tabular}

Figure 6: Number of significant genes for each test statistic with BH-FDR and SAM.

In the inference based on the permutations $(\mathrm{BH}-$ FDR) and the SAM, most of the genes found by the five statistics are in common (see Figure 6). The plots of the samples and the isotonic trend of four best genes that are found in all statistics and in both the permutations and the SAM approaches are presented in Figure 7, which have shown a significant increasing monotone trend.

\section{Discussion}

We have shown the use of the IsoGene package for dose-response studies within a microarray setting along with the motivating examples. For the analysis using the SAM procedure, it should be noted that the fudge factor in the $\bar{E}_{01}^{2}$ is obtained based on the approach for F-type test statistic discussed by Chu et al. (2001) and should be used with caution. The performance of such an adjustment as compared to the $t$-type test statistics has not yet been investigated in terms of power and control of the FDR. Therefore, it is advisable to use the fudge factor in the $t$-type test statistics, such as the $M$ and modified $M$ test statistics. 

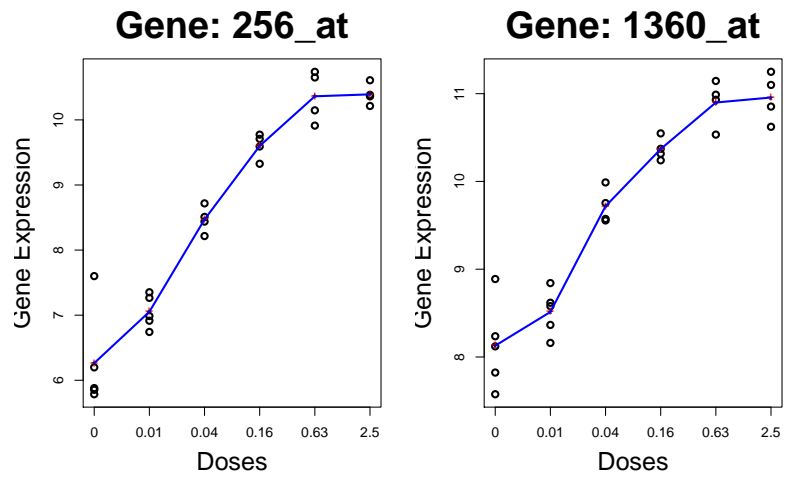

Gene: 924_at
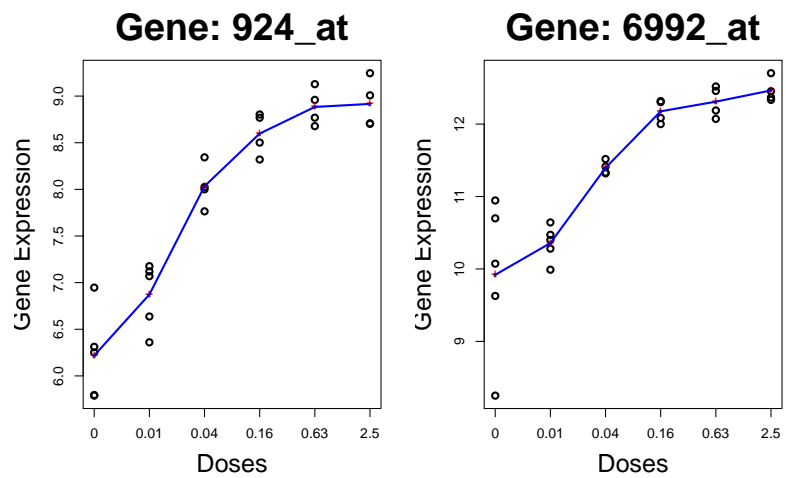

Figure 7: Plots of the samples (circles) and the isotonic trend (solid blue line) for the four best genes with a significant monotone trend.

The calculation of the raw $p$-values based on permutations using the IsoRawp () function is computationally intensive. For example, when we used 100 permutations for analyzing 10,000 genes, it takes around 30 minutes. When we use 1,000 permutations, the elapsed time increases around 10 times. Usually to approximate the null distribution of the test statistics, a large number of permutations is needed, which leads to the increase of computation time. Considering the computation time, we also provide the $p$-values obtained from joint distribution of permutation statistics for all genes which is implemented in function IsoTest $\operatorname{SAM}()$. This approach is sufficient to obtain small $p$-values using a small number of permutations. Alternatively, one can also use the SAM procedure which does not require a large number of permutations and takes less computation time. However caution should be drawn to the comparison of the SAM procedure with and without the fudge factor.

A further development of this package is the Graphical User Interface (GUI) using tcl/tk for users without knowledge of $\mathrm{R}$ programming.

\section{Acknowledgement}

Financial support from the IAP research network nr P5/24 of the Belgian Government (Belgian Science Policy) is gratefully acknowledged.

\section{Bibliography}

R. Barlow, D. Bartholomew, M. Bremner, and H. Brunk. Statistical Inference Under Order Restriction. New York: Wiley, 1972.

D. Bartholomew. Ordered tests in the analysis of variance. Biometrika, 48:325-332, 1961.

Y. Benjamini and Y. Hochberg. Controlling the false discovery rate: a practical and powerful approach to multiple testing. J. R. Statist. Soc. B, 57:289-300, 1995.

Y. Benjamini and D. Yekutieli. The control of the false discovery rate in multiple testing under dependency. ANN STAT, 29 (4):1165-1188, 2001.

G. Chu, B. Narasimhan, R. Tibshirani, and V. Tusher. SAM "Significance Analysis of Microarrays" users guide and technical document, 2001. URL http: //www-stat. stanford.edu/ tibs/SAM/ .

C. Chuang-Stein and A. Agresti. Tutorial in biostatistics: A review of tests for detecting a monotone dose-response relationship with ordinal response data. Statistics in Medicine, 16:2599-2618, 1997.

S. Dudoit and M. J. van der Laan. Multiple Testing Procedures with Applications to Genomics. Springer, 2008.

S. Dudoit, J. P. Shaffer, and J. Boldrick. Multiple hypothesis testing in microarray experiments. Statistical Science, 18 (1):71-103, 2003.

S. Falcon, M. Morgan, and R. Gentleman. An introduction to BioConductor's ExpressionSet class. a BioConductor vignette. Bioconductor Vignettes, URL http://bioconductor.org/packages/2.3/ bioc/vignettes/Biobase/inst/doc/

ExpressionSetIntroduction.pdf, 2007.

Y. Ge, S. Dudoit, and T. P. Speed. Resampling- based multiple testing for microarray data analysis. Test, 12 (1):1-77, 2003.

H. Göhlmann and W. Talloen. Gene Expression Studies Using Affymetrix Microarrays. Chapman \& Hall/CRC, 2009.

J. Hu, M. Kapoor, W. Zhang, S. Hamilton, and K. Coombes. Analysis of dose response effects on gene expression data with comparison of two microarray platforms. Bioinformatics, 21(17):35243529, 2005.

D. Lin, Z. Shkedy, D. Yekutieli, T. Burzykowski, H. Göhlmann, A. De Bondt, T. Perera, T. Geerts, and L. Bijnens. Testing for trends in dose-response microarray experiments: A comparison of several testing procedures, multiplicity and resamplingbased inference. Statistical Applications in Genetics and Molecular Biology, 6(1):Article 26, 2007. 
D. Lin, Z. Shkedy, D. Yekutieli, D. Amaratunga, and L. Bijnens., editors. Modeling Dose-response Microarray Data in Early Drug Development Experiments Using $R$. Springer, (to be published in 2010).

R. Marcus. The powers of some tests of the quality of normal means against an ordered alternative. Biometrika, 63:177-83, 1976.

J. Pinheiro, F. Bretz, and M. Branson. Analysis of dose response studies: Modeling approaches. pp. 146-171 in Ting, N. (ed.) Dose Finding in Drug Development. Springer, New York, 2006.

T. Robertson, F. Wright, and R. Dykstra. Order Restricted Statistical Inference. Wiley, 1988.

S. Ruberg. Dose response studies. i. some design considerations. J. Biopharm. Stat, 5(1):1-14, 1995a.

S. Ruberg. Dose response studies. ii. analysis and interpretation. J. Biopharm. Stat, 5(1):15-42, $1995 \mathrm{~b}$.

J. D. Storey and R. Tibshirani. Statistical significance for genomewide studies. Proceedings of the National Academy of Sciences, 100 no. 16:9440-9445, 2003.

V. Tusher, R. Tibshirani, and G. Chu. Significance analysis of microarrays applied to the ionizing radiation response. Proceedings of the National Academy of Sciences, 98:5116-5121, 2001.

D. Williams. A test for differences between treatment means when several dose levels are com- pared with a zero dose control. Biometrics, 27:103117, 1971.

D. Williams. The comparison of several dose levels with a zero dose control. Biometrics, 28:519-531, 1972.

Setia Pramana, Dan Lin, Philippe Haldermans and Ziv Shkedy

Interuniversity Institute for Biostatistics and Statistical Bioinformatics, CenStat, Universiteit Hasselt, Agoralaan 1, B3590 Diepenbeek, Belgium

setia.pramana@uhasselt.be

dan.lin@uhasselt.be

philippe.haldermans@uhasselt.be

ziv.shkedy@uhasselt.be

Tobias Verbeke

OpenAnalytics BVBA

Kortestraat 30A, 2220 Heist-op-den-Berg, Belgium

tobias.verbekedopenanalytics.be

Hinrich Göhlmann, An De Bondt, Willem Talloen and Luc Bijnens

Johnson and Johnson Pharmaceutical Research and Development, a division of Janssen Pharmaceutica, Beerse, Belgium

HGOEHLMA@PRDBE. jnj.com

ADBONDTQits.jnj.com

WTALLOENQits.jnj.com

LBIJNENSQits.jnj.com 